\title{
Development of a Reconfigurable Fixture for the Automated Assembly and Disassembly of High Pressure Rotors for Rolls-Royce Aero Engines
}

\author{
Thomas Papastathis ${ }^{1}$, Marco Ryll ${ }^{1}$, Stuart Bone ${ }^{2}$, and Svetan Ratchev ${ }^{1}$ \\ ${ }^{1}$ School of Mechanical, Materials and Manufacturing Engineering, \\ The University of Nottingham \\ \{epxtp, epxmr3, svetan.ratchev\} @nottingham.ac.uk \\ ${ }^{2}$ Rolls-Royce plc, Technology Acquisition Department \\ stuart.bonearolls-royce.co.uk
}

\begin{abstract}
This paper describes a novel fixturing system for the automated assembly and disassembly of aero engine components. The proposed system has the ability to automatically reconfigure for a number of different parts and allows the precise control and monitoring of its clamping positions and exerted clamping forces. Aspects of the control software of this system, like the graphical user interface (GUI) and the relational data base system containing postprocess and configuration data, are described.
\end{abstract}

\section{Introduction}

The manufacturing of aero engines and its sub-modules is traditionally characterised by rigorous product quality requirements and tight tolerances. Additionally, as a result of the global competition, increased product diversity is fast becoming a requirement for the aerospace sector too. At the same time, aero engine manufacturers, like Rolls-Royce, are adopting the Product Service System (PSS) paradigm. Because of the latter, manufacturers are not only working towards reducing lead times, but also minimising service and repair costs and efforts. For this reason, modern aeroassembly processes need to be upgraded by automated and rapidly reconfigurable manufacturing solutions in their production environment. Fixtures are a vital part of this environment which are devices designed to locate, support and hold workpieces in a desired position for an operation to be performed. In the past decades, industry and researchers have shown an increased interest in fixtures and their effect on the manufacturing outcome. In particular, a number of research work has addressed the issues of reconfigurable fixturing systems. Al-Habaibeh et al. [1] proposed a reconfigurable fixture based on an array of locator pins. Offering increased flexibility and distributed clamping load, these fixtures have been trialled on small and flexible aero components such as Nozzle Guide Veins (NGVs). However, locating precision issues and limited accessibility have limited their application on the shop floor. Chakraborty et al. [2, 3] have presented a flexible fixturing system for automobile engine cylinder heads which is able to identify and reduce the positioning error, thus increasing dimensional accuracy of the final product. Other reconfigurable fixturing 
solutions have been presented in [4-6]. However, these solutions have not been applied in industrial application.

Recently, in an attempt to further decrease workpiece deformation and thus improve the final product quality, a number of researchers have tried to actively adapt the clamping forces of the fixture to external influences [7]. Tao et al. [8, 9] have experimentally proven that clamping loads and workpiece deformation can be drastically reduced if the clamping forces are maintained to an ideal value during the machining process. Liao et al. [10] further investigated on the effects of clamping forces on the surface quality of machined automobile engine blocks. It was shown that clamping forces affect significantly the surface quality of the machined part, especially as the workpiece rigidity reduces. Despite the advantages of adaptive fixturing systems, this type of fixtures has seen limited transfer to industrial environments.

The review of the reported research indicates that a number of approaches exist for either reconfigurable fixturing or adaptive fixturing. However, the current solutions do not combine these two aspects into an integrated approach, the benefits of which could be significant, especially for industrial application. Additionally, the proposed solutions for adaptive fixtures appear to concentrate mainly on machining operations and are not adequately addressing the needs of aero assembly operations.

In an attempt to overcome these gaps and meet the future challenges of aero engine assembly within Rolls Royce plc., this paper reports on the ongoing development of a fixturing system for aero assembly components that is both reconfigurable and adaptive. The remaining paper is organized as follows. Section two describes the current process and future challenges for Rolls Royce. This is followed by an electromechanical system overview of the fixture in section three, while section 4 discusses aspects of the software architecture.

\section{Current Process Description}

The proposed fixturing system will be used for the assembly of the various stages of the High Pressure Compressor (HPC). This component sits in the heart of the jet engine (Fig. 1) and comprises various cylindrical stages, with diameters ranging from a few hundreds of millimetres to three thousand millimetres depending on the engine

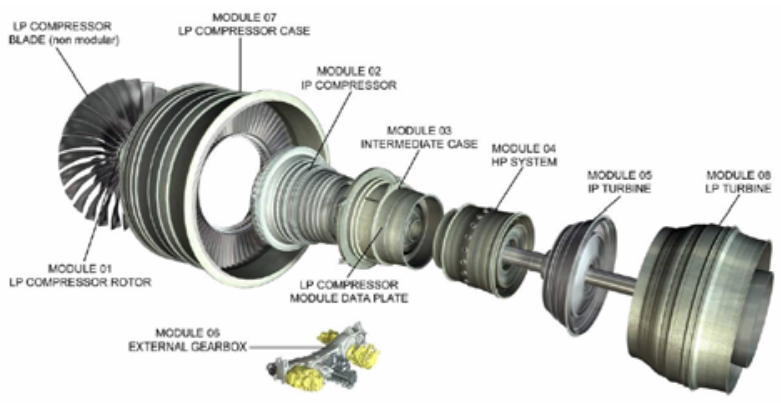

Fig. 1. Modular break-down of a Rolls-Royce Trent Engine 
model. Rotating at very high speeds and being exposed to enormous temperatures during operation, the HPC must conform to strict quality requirements regarding vibration, geometrical run-out and swash. On top of that, geometrical differences between the parts of the different engine variants demand fixture set up changes that elongate the overall lead time. These challenges in the assembly process of the HPC, are also present during disassembly for rework, service and repair purposes and subsequent re-assembly.

Currently, the assembly and disassembly process of HPCs is mainly manual, involving a series of activities performed by human operators. As an essential part of the operation, a hydraulic fixture is utilised to clamp the stages in the desired position for a predetermined amount of time. The latter is entirely operator controlled and its physical reconfiguration to accommodate the different part sizes, is carried out manually. Although this fixturing solution fully satisfies the present performance and process throughput demand, envisaged developments within Rolls-Royce predict the necessity to reduce lead times and further increase the repeatability of the fixturing outcome. Nevertheless, the current process could face three main challenges in the future. Firstly, the time-to-reconfigure and assembly/dis-assembly process cycle times characterising the current fixturing system could prove to be a bottleneck when looking to reduce production and servicing times. Manual reconfiguration could inhibit the necessary fast response when a setup change-over is requested. Speeding up the assembly and dis-assembly processes would also help reduce lead-times. Secondly, the currently acceptable percentage of parts failing the quality tests and demanding rework needs to be reduced as more parts are to be manufactured per year. This will keep repair and rework costs to a minimum. For this reason, a more repeatable process is necessary, thus the part of the fixturing cycle that is dedicated to manual labour needs to be reduced and the process parameters optimised and more tightly controlled. Finally, the ability to monitor clamping forces and generate automatic post-production reports, which is not available in the current fixturing system, could help identify performance variations and assist in adapting the process more effectively to future requirements.

\section{Design Overview of Fixturing System}

A simplified overview of the proposed fixture is shown in figure 2. The proposed fixture, developed in cooperation with ETN Aviation GmbH, comprises of 6 fixturing elements, i.e. three clamps and three support elements. The upper three elements are used to provide clamping and separating forces to the upper stages, whilst the three support elements are used to simply locate and hold the lower stages during the disassembly process. In total the fixture deploys three direct current (DC) servomotor actuators and nine off-the-shelf step motor rotary actuators on a circular frame to allow repositioning of the clamps in radial and vertical direction.

These actuators grant the fixture the ability to automatically reconfigure for a number of different cylindrical parts. In detail, three DC ToX ElectricDrive EPMK electromechanical actuators are used in a vertical orientation to provide the clamps with the capability to clamp the stages together during the assembly process with a specified amount of force and over a predefined period of time. Additionally, in case 


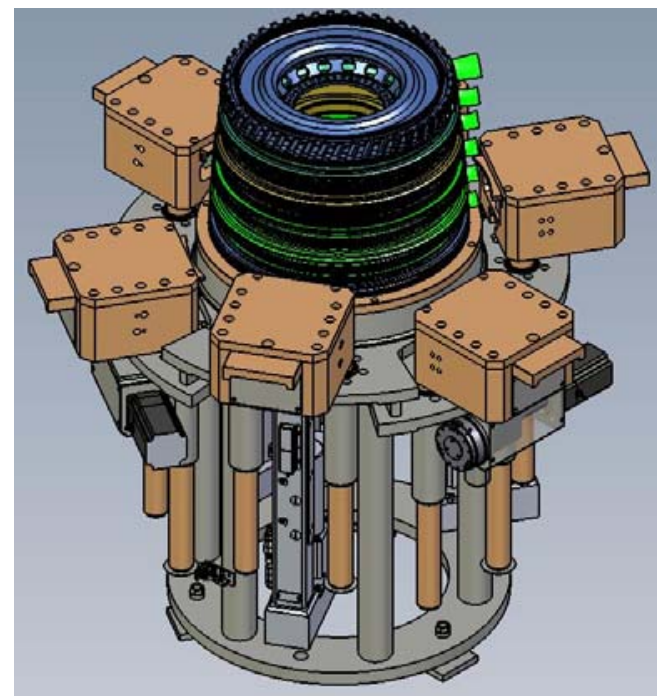

Fig. 2. Simplified design overview of the fixturing system

of servicing and repair activities, they are used to disassemble the HPC by pushing the stages apart. The actuators are controlled by three separate ToX L9400 X-Line controllers. Communication of the controllers is established via ProfiBus, ensuring tight control of actuation times to achieve synchronised clamping. Individual actuation of each clamp is also possible. The actuators are equipped with force feedback through strain gauges and position feedback via encoders. These provide the necessary information for maintaining stable clamping forces over the process cycle and for reporting purposes at the end of the cycle. Feedback signals are also transferred to a PC via ProfiBus.

Three NanoTech AD8918 stepper motors driving an equal number of ball screws are used to actuate vertically the support elements. Finally, six stepper motor based linear actuators are used to achieve radial movement of both the clamps and the support elements. All nine stepper motor are powered via an equal number of SMCI47 stepper drivers. Communication of the PC to these drivers is achieved through an Advantech PCI-1758U I/O card. An array of ten Balluf BESM08EHPSC15B-S04 proximity sensors is used to reference the system at startup.

The use of electromechanical actuation and the combination of the previously mentioned actuator types produces a compact and clean solution with only marginally higher cost than an equivalent hydraulic solution. Nevertheless, this design gives the extra advantage of allowing maximum control over process parameters such as actuating forces, clamp actuation sequence and clamp positions. The latter is especially important for the automatic reconfiguration of the fixture for different part sizes.

\section{Overview on the System Software}

Operators can control the operation of the fixture through a PC-based control environment. The latter consists mainly of three components: (1) a data base system storing all 
relevant process parameters for each workpiece and for both the assembly and disassembly; (2) a graphical user interface guiding the operator through reconfiguration sequence and (3) a series of communication interfaces to the controllers of the actuators on which the force profiles of the clamps are stored. When a new workpiece is detected, the control software can retrieve the adequate process parameters from the data base and subsequently trigger the related clamping profiles on the actuators. For this purpose, the software communicates with the actuator controllers of the fixture via a ProfiBus interface and digital I/O connections as described in the previous section. During the process the software monitors the online force and position feedback from the clamps and stores all relevant data permanently on the data base. This information can be used to generate post-production reports, which is realised with the LabView Diadem Reporting functionally. An overview on the entire system is provided in figure 3.

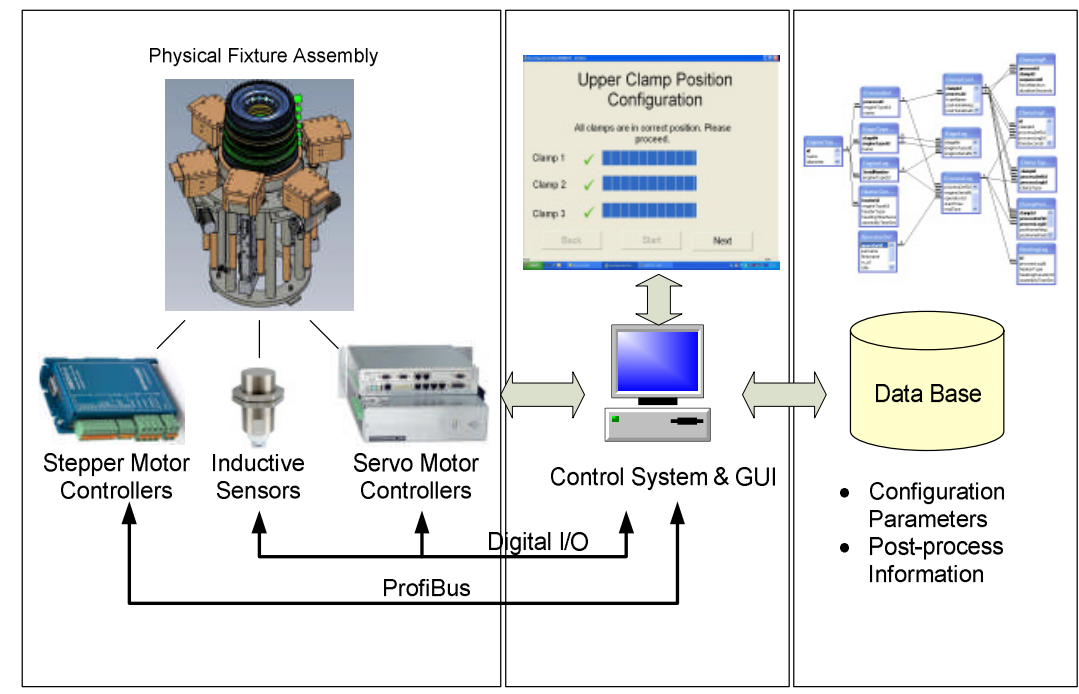

Fig. 3. Overview of the software and communication system

In the following sections, important aspects of the data base structure and the user interface are briefly described. The communication interfaces and routines on the actuator controllers shall be omitted here for brevity.

\subsection{Data Base Design and Implementation}

An entity-relationship model (ERM) has been developed to represent the relations and characteristics of the various engine types, processes, fixture configuration parameters and post-process data. This model has been implemented with the Microsoft Access database system. Key to this model is the table EngineTypeDef, which holds information about the various engine types, including an identifier and a name. Linked to this table via foreign keys, the table StageTypeDef contains information about the structure of an engine type while the available processes for a certain engine type are defined in the table ProcessDef. Each process is defined by a numerical id, a name and a 
link to the engine type table (foreign key). This allows the grouping of the required fixture configuration parameters for a particular engine/process pair in the table ClampConfiguration, which contains the clamping positions, home positions, desired force profiles etc. Each record in this table is uniquely identified by a so-called primary key, consisting of a numerical clamp id and the process id, which links back to the ProcessDef table. Consequently, the data model can be easily adapted to future fixture designs because an arbitrary number of clamps can be defined for each process. When the system is in operation, a record for the particular engine and process is created in the data base tables EngineLog and ProcessLog. The former contains a serial number and is linked to the EngineTypeDef table. The latter is linked to the ProcessDef table and EngineLog and holds global information of the particular process such as start time, end time and a link to the operator. More detailed process information, like the actual measured forces of a particular clamp, the actual installed clamp types and the measured clamp positions, are stored in separate tables that are linked via foreign keys to the ProcessLog table. This structure allows to easily extend the system in the future, should the monitoring of other process parameters become necessary.

\subsection{Graphical User Interface}

To guide the operator through the various processes, a Graphical User Interface has been developed using the programming language $\mathrm{C}++$ and the Microsoft Foundation Classes (MFC). The software relies on an object-oriented approach where each reconfiguration step is modelled as one class according to the object-oriented Command design pattern [11]. In this design pattern, a single abstract root class defines a common interface that consists only of the method execute(). Based on this, a variable number of child classes can be extended from the super class to implement the execute()-method differently. The execute()-method does not have any parameters, because all information necessary to execute the task of a particular command is encapsulated as attributes when the command-object is created.

There are several advantages of this design pattern which are all based on the common interface defined in the abstract parent class. First and foremost, this approach allows easy enhancement of the system with new reconfiguration tasks. If in the future the necessity of further reconfiguration steps evolves, the class hierarchy can easily be extended without affecting the rest of the system. Secondly, decomposing the entire fixture reconfiguration into atomic steps modelled as single objects significantly simplifies the complexity for control software. In more detail, due to the common interface, the execution of the entire reconfiguration sequence is reduced to simple calls of the execute()-method of each command.

\section{Conclusions}

In this paper, hardware and software aspects of a new fixturing system for the automated assembly of aero-engines has been described. The system is addressing three major requirements of future aero-assembly, namely the reduction of reconfiguration times, higher repeatability due to better process control and the ability to monitor process parameters and generate post-process reports. The fixturing hardware consists 
of three DC-servomotor actuators that generate the clamping forces and a series of stepper motors responsible for the positioning. Key to the control software is a graphical user interface, that guides the operator through the reconfiguration sequences and a relational data base system, holding important process and configuration data.

Acknowledgments. The reported research is conducted as part of the ongoing European Commission FP6 funded integrated project (FP6-2004-NMP-NI-4) - AFFIX "Aligning, Holding and Fixing Flexible and Difficult to Handle Components". The financial support of the EU and the contributions by the project consortium members are gratefully acknowledged.

\section{References}

1. Al-Habaibeh, A., Gindy, N., Parkin, R.M.: Experimental Design and Investigation of a pin-type reconfigurable clamping system for manufacturing aerospace components. Journal of Engineering and Manufacture - Proceedings of the Institution for Mechanical Engineers Part B 217, 1771-1777 (2003)

2. Chakraborty, D., De Meter, E.C., Szuba, P.S.: Part location algorithms for an intelligent fixturing system part 1: system description and algorithm development. Journal of Manufacturing Systems 20, 124-134 (2001)

3. Chakraborty, D., De Meter, E.C., Szuba, P.S.: Part location algorithms for an Intelligent Fixturing System Part 2: algorithm testing and evaluation. Journal of Manufacturing Systems 20, 135-148 (2001)

4. Chan, K., Benhabib, B., Dai, M.: A reconfigurable fixturing system for robotic assemble. Journal of Manufacturing Systems 9, 206-221 (1990)

5. Du, H., Lin, G.C.I.: Development of an automated flexible fixture for planar objects. Robotics and Computer-Integrated Manufacturing 14, 173 (1998)

6. Youcef-Toumi, K., Buitrago, J.H.: Design and implementation of robot-operated adaptable and modular fixtures. Robotics \& Computer-Integrated Manufacturing 5, 343-356 (1989)

7. Nee, A.Y.C., Tao, Z.J., Kumar, A.S.: An Advanced Treatise on Fixture Design and Planning. World Scientific Publishing, Singapore (2004)

8. Tao, Z.J., Kumar, A.S., Nee, A.Y.C.: Automatic generation of dynamic clamping forces for machining fixtures. International Journal of Production Research 37, 2755 (1999)

9. Tao, Z.J., et al.: Modelling and experimental investigation of a sensor-integrated workpiece-fixture system. International Journal of Computer Applications in Technology 10, 236-250 (1997)

10. Liao, Y.G., Hu, S.J.: An integrated model of a fixture-workpiece system for surface quality prediction. International Journal of Advanced Manufacturing Technology 17, 810-818 (2001)

11. Gamma, E., et al.: Design Patterns. Elements of Reusable Object-Oriented Software. Addison-Wesley Longman, Amsterdam (1995) 